\title{
THE CORONA STABILIZATION SYSTEM OF A VAN DE GRAAFF GENERATOR
}

\author{
A. VERMEER and B. A. STRASTERS
}

Fysisch Laboratorium, Rijksuniversiteit, Sorbonnelaan 4, Utrecht 2506, The Netherlands

\begin{abstract}
Résumé. - Le système de stabilisation par décharge corona d'un Van de Graaff Tandem EN a été étudié afin de pouvoir définir son fonctionnement dans la réduction des fluctuations rapides. Avec les paramètres mesurés de la triode corona, à savoir, $\mu, R_{\mathrm{i}}$ et le temps de transition, un circuit électrique équivalent a été formé. De ce circuit on peut calculer un facteur de stabilisation $S$. Les valeurs théoriques du facteur $S$ correspondent assez bien aux valeurs expérimentales.
\end{abstract}

Abstract. - The corona stabilization system of an EN Tandem Van de Graaff accelerator is studied in order to reduce the fast fluctuations. With the measured parameters of the corona triode, i.e. $\mu, R_{\mathrm{i}}$ and the transit time, an electrical replacement diagram is given. From this diagram a stabilizing factor $S$ can be calculated. A comparison between the theoretical and experimental values for $S$ shows a good agreement.

In a previous paper the fluctuations in the charge transport system of an EN Tandem Van de Graaff, which contribute to the terminal ripple, have been investigated [1]. To reduce the terminal ripple of a Van de Graaff generator, a corona triode stabilization system is generally used. We did a research into the stabilization system, in order to understand its properties and to optimize it. Because the corona triode itself is an essential part of the system, we looked to the corona triode in more detail.

In the practical set-up the corona current is delivered by a field emission cathode, which consists of a number of sharp needles. The anode of the corona discharge is the terminal electrode. A mushroom shaped electrode around the corona needles functions as a grid. This grid is normally at earth potential. The whole, i.e. the corona needles, the mushroom and the terminal form a triode of which the properties can be described in the same way as for a triode tube. The three coefficients of a triode are: the internal resistance $R_{\mathrm{i}}$, the mutual conductance $S$, and the amplification factor $\mu$. The three coefficients are interrelated via the formula known as the Barkhausen formula: $\mu=R_{\mathrm{i}} S$.

A lot of measurements were done to obtain these parameters under different experimental conditions. In figure 1 an example of such a measurement is shown. Here the internal resistance $R_{\mathrm{i}}$ is plotted at a constant corona current of $50 \mu \mathrm{A}$, as a function of the distance $d_{\mathrm{ac}}$ between the corona needles and the terminal. In the graph the relevant terminal voltages are also given.

The fourth parameter of the corona triode, i.e. the transit time, will now be considered. The transit time

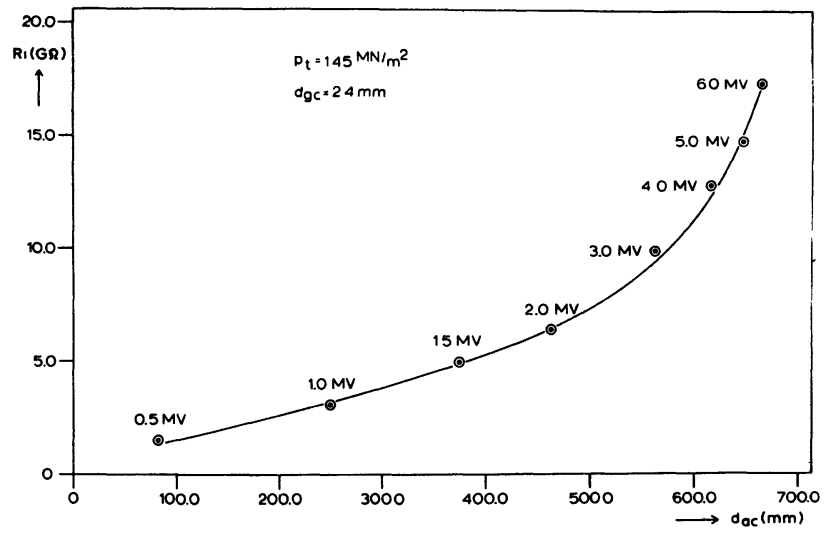

FIG. 1. - The internal resistance $R_{\mathrm{i}}$ is plotted as a function of the distance from the corona needles to the terminal $d_{\mathrm{ac}}$. The corona current is kept constant at $50 \mu \mathrm{A}$.

$d_{\mathrm{gc}}=$ the protruding needle length of the corona needles.

$P_{\mathrm{t}}=$ the tank gas pressure.

of the corona discharge is the time the charge carriers need to cross the discharge. The transit time is caused by the enertia of the charge carriers and gives an additional phase shift between the grid and terminal voltage. The transit time can be measured in the following way. A.l.f. sinusoidal signal is put on the grid of the regulating triode 6BK4 (see Fig. 2). The phase shift $\varnothing_{\text {tr }}$ is measured with a storage scope, between the signal of the pick-up amplifier and the signal on the anode of the 6BK4.

A storage scope is used because the sinusoidal signal is superposed on the terminal ripple. With a storage scope the form of the terminal signal is easy to average. The frequency response of the used terminal pickup amplifier is flat from $0.1-200 \mathrm{~Hz}$. 


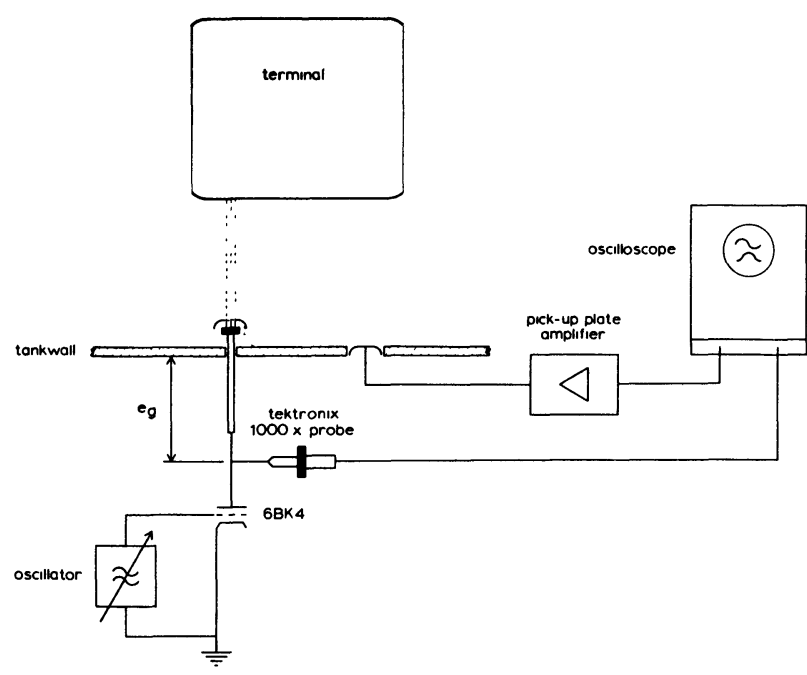

FIG. 2. - The circuit for the transit time measurements.

In figure 3 a picture of both signals is shown. From the phase shift $\varnothing_{\mathrm{tr}}$ one can calculate the transit time $t_{\mathrm{tr}}=\frac{\Delta \varnothing_{\mathrm{tr}}}{f .360^{\circ}}$, at which $\Delta \varnothing_{\mathrm{tr}}=\varnothing_{\mathrm{tr}}-90^{\circ}$ and $f$ is the frequency in hertz. To obtain a higher accuracy, the measuring frequency is choosen $60 \mathrm{~Hz}$. From the measured phase shift $\varnothing_{\mathrm{tr}}, 90^{\circ}$ must be subtracted, which is the contribution to the phase shift due to the terminal capacity $C_{\mathrm{t}}$ (see Fig. 5). The assumption is made that the transit time $t_{\mathrm{tr}}$ is independent of the frequency, and of the corona current, which is not
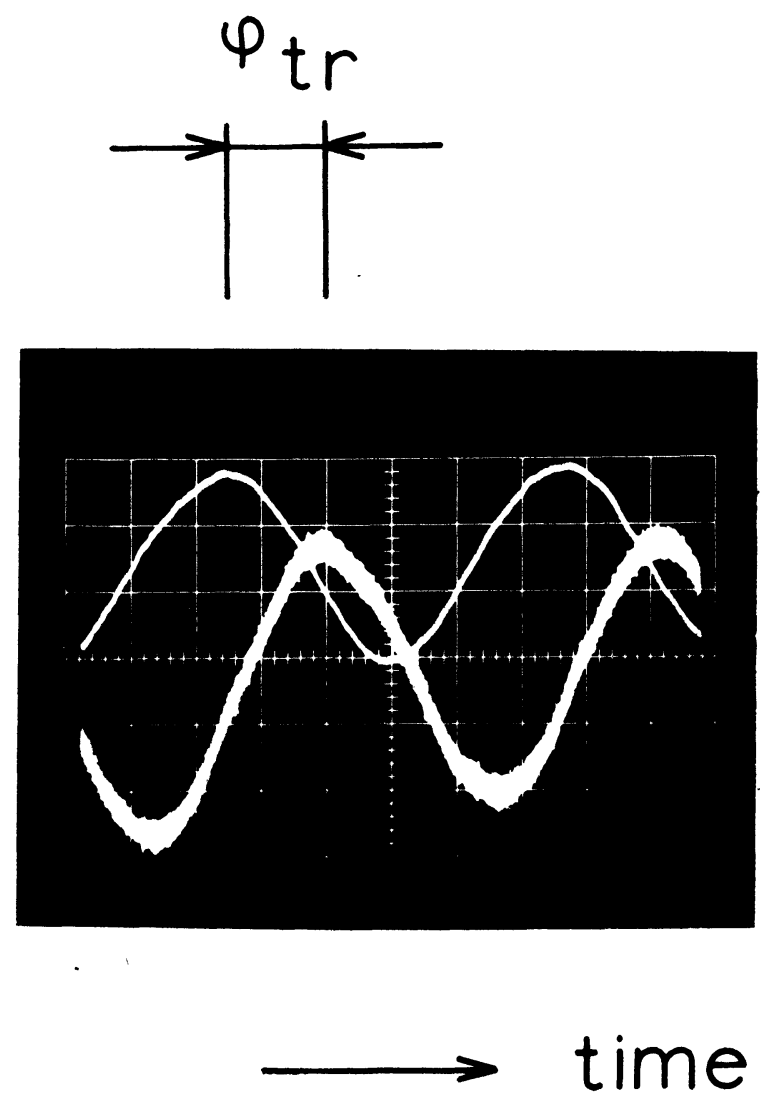

Fig. 3. - The phase shift $\varnothing_{\mathrm{tr}}$ between the grid signal of the corona triode (upper) and the terminal signal (lower). The measuring frequency is $60 \mathrm{~Hz}$. unreasonably. In figure 4 the results of these measurements are shown.

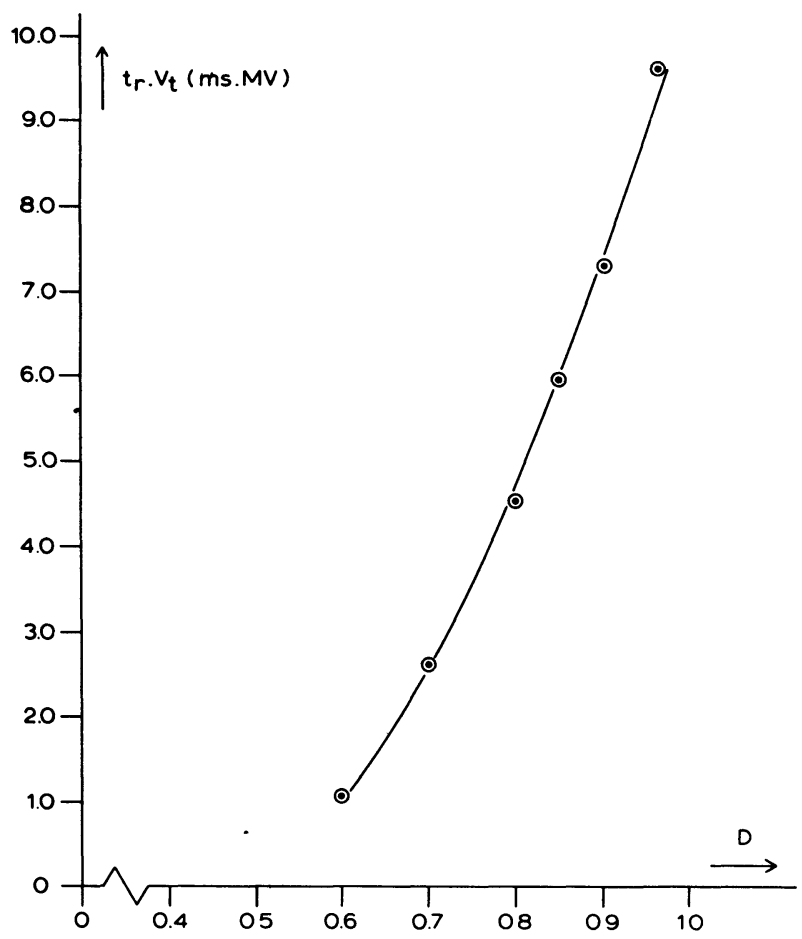

Fig. 4. - The product of the transit time and the terminal voltage is plotted as a function of the distance parameter $D$. The distance parameter $D$ is defined as the distance between the corona points and the tanks axis, divided by the tank radius.

Because the drift velocity of the charge carriers in the corona discharge is a function of the terminal voltage, in figure 4 the product is plotted of the transit time $t_{\mathrm{tr}}$ and the terminal voltage. As abscissa the distance between the corona needles and the terminal is taken. This distance is expressed in the dimensionless quantity $D$ which is the distance from the corona needles to the tank axis divided by the internal tank radius. It is possible to translate this phase shift into an electrical circuit element, as a selfinductance. This selfinductance can be thought in series with the already mentioned internal resistance $R_{\mathrm{i}}$ of the corona triode. In figure 5 the substitution diagram for the

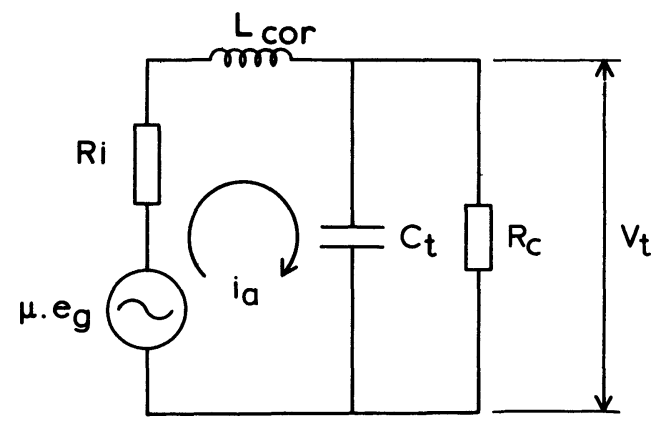

FIG. 5. - The equivalent circuit for the corona triode. $\mu=$ the amplification factor of the corona triode. $e_{\mathrm{g}}=$ the grid voltage.

$e_{\mathrm{g}}=$ the terminal voltage.
$V_{\mathrm{t}}=$ the

$C_{\mathrm{t}}=$ the terminal capacity.

$R_{\mathrm{c}}=$ the column resistance. 
corona triode is drawn. Herewith the corona triode is presented by a voltage source with an EMF of $\mu e_{\mathrm{g}}$ in series with its internal resistance $R_{\mathrm{i}}$ and a self inductance $L_{\text {cor }}$. The terminal capacity $C_{\mathrm{t}}$ and the column resistance $R_{\mathrm{c}}$ form the external load of this circuit. The influence of the column resistance can be neglected, because of its very high value compared to the reactance of the terminal capacity. The selfinductance $L_{\text {cor }}$ can be calculated from the measured phase shift $\Delta \varnothing_{\mathrm{tr}}=\omega t_{\mathrm{tr}}$ between the grid voltage $e_{\mathrm{g}}$ and the terminal voltage $V_{\mathrm{t}}$. The value of this selfinductance $L_{\text {cor }}$ for a given frequency $\omega$, is given by:

$$
L_{\mathrm{cor}}=\frac{\left(1+\omega^{2} R_{\mathrm{i}}^{2} C_{\mathrm{t}}^{2}\right) \operatorname{tg} \omega t_{\mathrm{tr}}}{\omega^{2} C_{\mathrm{t}}\left(\omega R_{\mathrm{i}} C_{\mathrm{t}}+\operatorname{tg} \omega t_{\mathrm{tr}}\right)}
$$

In figure 6 the calculated selfinductance $L_{\text {cor }}$ is plotted as a function of the frequency for different values of the transit time.

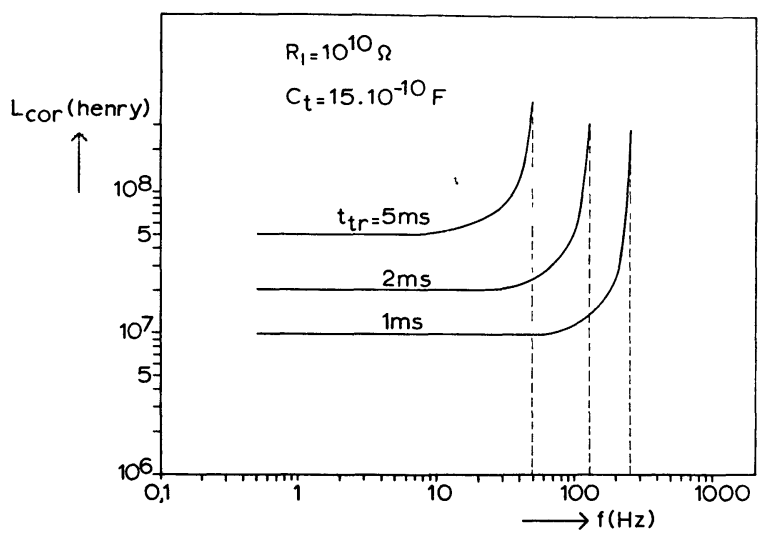

FIG. 6. - The calculated value of the corona selfinductance $L_{\text {cor }}$ for different transit times as a function of the frequency.

The transit time $t_{\mathrm{tr}}$ is found from figure 4 by dividing the values of the ordinate by the terminal voltage $V_{\mathrm{t}}$. In practice the value of the terminal voltage $V_{\mathrm{t}}$ shall be always adapted to the value of the distance parameter $D$. That means that at a short corona discharge (low $D$ ) the terminal voltage $V_{\mathrm{t}}$ shall be low and also the reverse. One finds for an EN tandem accelerator transit times between 1 and $3 \mathrm{~ms}$. The obtained data of the parameters of the corona triode in the preceding section can be used at the study of the corona stabilization system. For the steering of the corona triode use is made of the regulating triode $6 \mathrm{BK} 4$, which is in series with the corona triode. Both triodes form together what is called a cascode circuit, as is described in the literature [2].

In the equivalent circuit of figure 7 , the main components of the system are indicated schematically. In the caption the different components of the circuit are described. The reference signal is taken from the pickup plate. The pick-up plate forms with the terminal electrode and the tank wall a capacitive voltage divi- der, which is frequency independent. The frequency region for which the stabilizer is designed, is from the

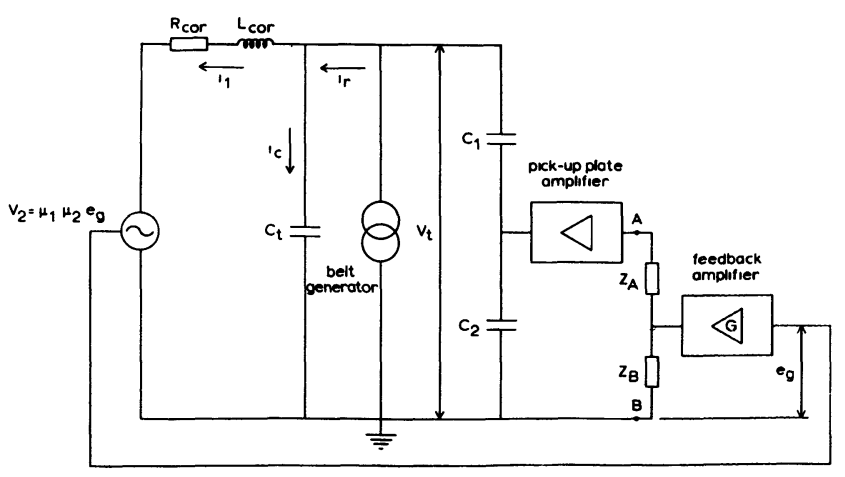

FIG. 7. - The equivalent circuit of the corona stabilization system.

$\mu_{1}=$ the amplification factor of the triode $6 \mathrm{BK} 4$

$\mu_{2}=$ the amplification factor of the corona triode.

$i_{\mathrm{r}}=$ the ripple current from the belt generator.

$C_{1} / C_{2}=$ the pick-up plate voltage divider.

$Z_{\mathrm{A}} / Z_{\mathrm{B}}=$ the correcting network.

belt frequency (about $2.5 \mathrm{~Hz}$ ) up to $45 \mathrm{~Hz}$. In this range the frequencies of the belt system are found as an earlier research shows [1]. At a Van de Graaff generator charge is transported to the terminal capacitor by means of the belt, which acts as a current source with a very high internal resistance. The ripple current $i_{\mathrm{r}}$, which is superimposed on the DC current, can therefore be thoughed to be delivered by a ACcurrent source.

The feedback network consists of the impedances $Z_{\mathrm{A}}$ and $Z_{\mathrm{B}}$, which will be discussed further on. A formula will be derived for the stabilization factor $S$ with the help of the circuit of figure 7. The factor $S$ is given as the ratio between the amplitudes of the terminal voltage fluctuations, without and with the stabilizer switched on. The factor $S$ shows to be a function of the frequency $\omega$. For this circuit the following equations hold, as the current $i_{\mathrm{r}}$ is delivered by a current source (belt generator) and the starting conditions are put zero

$$
\begin{gathered}
\mu_{1}\left(\mu_{2}+1\right) e_{\mathrm{g}}=i_{1} R_{\mathrm{i}}+L_{\mathrm{cor}} \frac{\mathrm{d} i_{1}}{\mathrm{~d} t}-\frac{1}{C_{\mathrm{t}}} \int_{0}^{\mathrm{t}} i_{\mathrm{c}} \mathrm{d} t \\
i_{\mathrm{r}}=i_{1}+i_{\mathrm{c}} \\
e_{\mathrm{g}}=\frac{Z_{\mathrm{B}}}{Z_{\mathrm{A}}+Z_{\mathrm{B}}} G \beta V_{\mathrm{t}}
\end{gathered}
$$

with $G$ being the amplification of the feedback amplifier. $\beta=\frac{V_{\mathrm{AB}}}{V_{\mathrm{t}}}, \beta$ is frequency independent in the considered frequency range.

Differentiation of equation (1) and substitution of $i_{\mathrm{r}}$ and $e_{\mathrm{g}}$ from equation (2) and equation (3) in the 
obtained equation, delivers after Laplace transformation:

$$
V_{\mathrm{t}_{\mathrm{p}}}=\frac{p+a}{p^{2}+p a+c\left\{1+\frac{G^{\prime} Z_{\mathrm{B}_{\mathrm{p}}}}{Z_{\mathrm{A}_{\mathrm{p}}}+Z_{\mathrm{B}_{\mathrm{p}}}}\right\}} \frac{l_{\mathrm{r}_{\mathrm{p}}}}{C_{\mathrm{t}}}
$$

Putting $a=R_{\mathrm{i}} / L_{\text {cor }}, c=1 / L_{\text {cor }} C_{\mathrm{t}}$ (index for p-region) and $\mathrm{G}^{\prime}=\mu_{1}\left(\mu_{2}+1\right) G$

By determining the ratio of the terminal ripple at the same $i_{r_{\mathrm{p}}}$, in the case the feedback is switched off and $o n$, one finds the stabilizing factor $S$. The first case one gets by putting $G^{\prime}$ equal to zero. In this way the stabilizing factor $S_{\mathrm{p}}$ in the p-region will be found as:

$S_{\mathrm{p}}=\frac{\left(V_{\mathrm{t}_{\mathrm{p}}}\right) G^{\prime}=0}{V_{\mathrm{t}_{\mathrm{p}}}}=1+\frac{c\left(1+G^{\prime} \frac{Z_{\mathrm{B}_{\mathrm{p}}}}{Z_{\mathrm{A}_{\mathrm{p}}}+Z_{\mathrm{B}_{\mathrm{p}}}}\right)}{p^{2}+p a+c}$

By choosing different impedances in the network $Z_{\mathrm{A}} / Z_{\mathrm{B}}$, the values of $S$ as a function of the frequency can be influenced. In the frequency spectrum of the terminal ripple the lower frequency components are the strongest [1]. So a network configuration can be favourable at which the low frequency components are the strongest reduced. At every configuration, one must look if the requirements of the Routh-Hurwitz criterion of stability are fulfilled [3]. Otherwise, the circuit becomes unstable and will oscillate. By substituting $p=j \omega$, in the formula for $S_{\mathrm{p}}$, one gets the stabilization factor $S$ as a function of the frequency. The stabilization factor can be measured using the analysing equipment described in reference [1]. The result of such a measurement, for the parameters given in the caption, is plotted in figure 8 .

For the same experimental conditions, the values of the factor $S$ are also calculated and plotted in the same figure. The theoretical and experimental curves are in a good agreement. In principle it is possible to obtain much higher values for the stabilizing factor $S$, by taking a high value for the loop gain $G$. However, the regulating triode $6 \mathrm{BK} 4$ is a limiting factor, because of its small grid space.

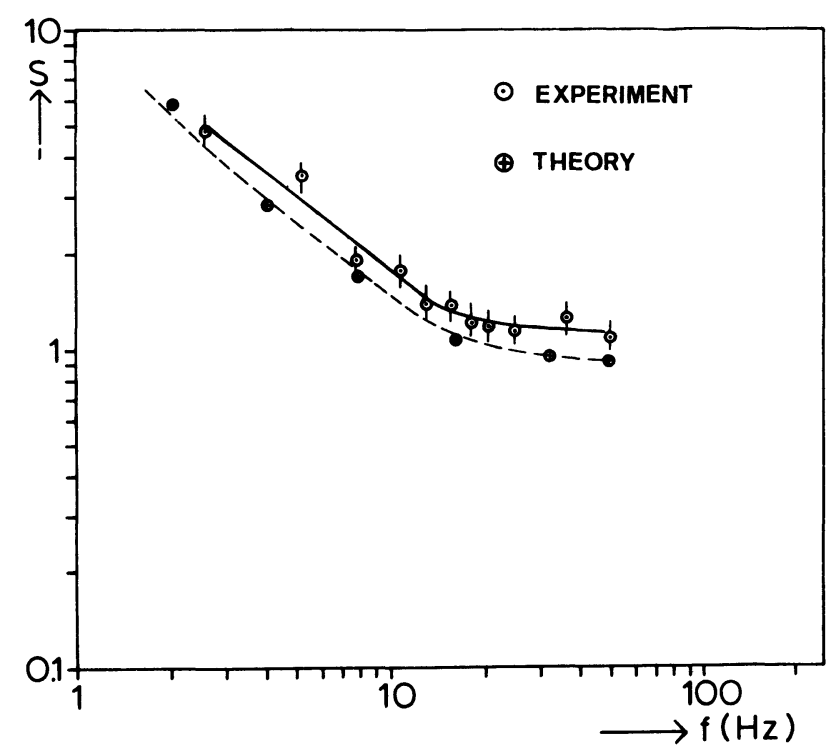

FIG. 8. - The experimental and theoretical stabilization factor $S$ as a function of the frequency, for the following conditions: $\mu_{1}=1000, \mu_{2}=20, \beta=5 \times 10^{-5}, G=100, L_{\text {cor }}=2 \times 10^{7} \mathrm{H}$, $C_{\mathrm{t}}=150 \mathrm{pF}, Z_{\mathrm{A}} / Z_{\mathrm{B}}=$ resistance network.

\section{References}

[1] Vermeer A. and Strasters B. A., Nucl. Instrum. Methods. 131 (1975) 213.

[2] De WaARD H. and Lazarus D., Modern Electronics (AddisonWesley Publishing Comp), 1966 p. 201.

[3] Taylor P. L., Servomechanisms, (Longmans), London, 1965 p. 302. 